\title{
A Strategy for Emergency Vehicle Preemption and Route Selection
}

\author{
Khaled Shaaban ${ }^{1}$ (D) Muhammad Asif $\mathrm{Khan}^{2} \cdot$ Ridha Hamila $^{2} \cdot$ Mohammad Ghanim $^{3}$
}

Received: 11 June 2018 / Accepted: 10 May 2019 / Published online: 17 May 2019

(c) The Author(s) 2019

\begin{abstract}
Emergency vehicle preemption (EVP) aims to give right of way to emergency vehicles (EV) heading toward the incident location through a network of signalized intersections by creating a green wave en-route. The design goals of EVP systems are two folds: first, to avoid any hindrance to the passage of EV along the road and at the intersections and second, to reduce the negative impact of preemption on general traffic. The negative impact of EVP on normal traffic can be minimized by selecting appropriate preemption strategy. The EVP schemes proposed earlier aim to minimize the travel time of the EV with no or little consideration to the negative impact of EVP on the normal traffic. In this study, a joint strategy for optimal path selection and EV preemption is developed. The proposed scheme selects the optimal path for the EV before it departs from its origin and then activates the preemption on each intersection en-route at the right time to clear the intersection before the EV reaches. The proposed EVP scheme also aims to minimize the impact of EVP over normal traffic at both stages (i.e., path selection phase and preemption phase). A major advantage of the proposed method is that once the optimal path is selected, the emergency information can be disseminated to other vehicles using vehicle-to-vehicle and vehicle-to-infrastructure communication in the $\mathrm{EV}$ path to clear the entire route or the approaching lane. The strategy was tested using a microscopic simulation environment for a real traffic network. The findings indicated a major reduction in the travel time of the EV while minimizing the impact of preemption on the normal traffic. The proposed strategy and evaluation procedure can be helpful for corresponding agencies and practitioners to assess the impact of implementing preemption on existing or proposed arterials.
\end{abstract}

Keywords Adaptive control · Traffic signal · Travel time $\cdot$ Vehicle detection

\section{Introduction}

Emergency vehicles (EVs) like fire trucks, ambulances, and police vehicles have the highest priority on roads to respond to an incident. To ensure free and uninterrupted travel to EVs

Khaled Shaaban

kshaaban@qu.edu.qa

Muhammad Asif Khan

mkhan@qu.edu.qa

Ridha Hamila

hamila@qu.edu.qa

Mohammad Ghanim

mghanim@birzeit.edu

1 Department of Civil Engineering/Qatar Transportation and Traffic Safety Center, Qatar University, PO Box 2713, Doha, Qatar

2 Department of Electrical Engineering, Qatar University, PO Box 2713, Doha, Qatar

3 Department of Civil and Environmental Engineering, Birzeit University, PO BOX 14, Birzeit, Palestine along its route to the incident location, traffic signal controllers deploy preemption strategies, benefiting those EVs. Emergency vehicle preemption system (EVP) interrupts the normal traffic signal timing operations in both isolated and coordinated signalized intersections by providing a green band to the EVs along its routes. EVP enables EVs to pass without stopping or waiting at intersections which would potentially reduce travel time and reduce conflicts with other vehicles in the system [1]. Similar strategies are used for rail preemption and transit signal priority [2]. However, it may also produce a negative impact on the general traffic not only at the same intersection where the EV is approaching but also on other neighboring intersections, especially in the case of coordinated signal control $[3,4]$.

Most of the signal preemption strategies are focused on isolated intersections. This kind of signal preemption strategy, which is based on local detection and clears the intersection one by one, can start only after an EV is detected and usually leads to inevitable intersection delay [5-7]. Moreover, when multiple signal preemptions are implemented 
during the peak period, the queue and delay of general traffic in the network will be negatively affected [8]. Implementing signal preemption from the perspective of an entire route can reduce the response time of EV. Although substantial progress has been made in signal preemption of an isolated intersection, research of dynamic signal preemption based on the entire route has dragged less attention. This study describes a proposed strategy for efficient preemption along a corridor and evaluates the impact of implementing the preemption strategy on a major arterial on both EVs and general traffic.

\section{Literature Review}

\subsection{System Description}

EV's preemption is employed by most modern traffic signal control systems such as SCATS [9], SCOOT [10], RHODES [11], and ACS Lite [12]. The working mechanism may differ in different systems, but the ultimate goals are the same, to decrease the EV travel time by avoiding stopping, reducing the negative impact of preemption on general traffic, and ensuring the safety of the vehicles, including EVs. The EVP system usually consists of the EV, a signal emitter, a signal detector, and a traffic signal controller. The process of preemption is initiated when an EV switches on the emergency button installed inside in the EV. The emitter installed at EV transmits the emergency signal to the signal detector located at all the traffic signals. The signal detector is actuated, and it requests the signal controller to give priority to the approaching EV at the given intersection. The signal controller interrupts the normal phase cycle and switches to green light to allow the traffic to flow at the particular approach of the EV arrival to avoid stopping. Several preemption systems exist including OPTICOM [13], BLISS [14], STROBECOM [15], and MIRT [16].

The OPTICOM priority control system is used worldwide for selected vehicles, including EVs. Initially, the system used an infrared emitter mounted on the EV that sends frequency coded optical messages to the detector mounted on the signal. The detector decodes the signal and sends the information to the phase selector and the phase selector then informs the signal controller to either extend the green cycle or skip the red cycle accordingly. OPTICOM is currently available with GPS and acoustic-based EV detection and tracking. BLISS is a centralized system with a single PC controlling up to 63 sets of traffic signals. Signal timing plans are calculated using TRANSYT. The EV is assigned with a vehicle identification (VID) tag. The controller, when receiving a request from EV with the VID attached, extends the green cycle according to the requirement. STROBECOM and MIRT are more or less similar to OPTICOM and use the optical emitter and detector.

The EVP function mainly depends on two phenomena: i) vehicle detection technique to detect the $\mathrm{EV}$ at the appropriate time and distance with the provision of speed and position information and traffic status on the EV route and ii) the preemption strategy to effectively utilize the green cycle of all intersections en-route.

\subsection{Vehicle Detection}

In the EVP system, vehicles are differentiated into general vehicles and EVs where the position, speed, count, and lane occupancy of vehicles are significant parameters. For the optimum preemption strategy, the signal controller must detect the EV in advance. The controller should detect the $\mathrm{EV}$ before a minimum time (detection time) that is required to clear the approaching lane before the arrival of the EV to avoid stopping of EV. The detection time depends upon the traffic conditions at the intersection. The controller should also know the current location of the EV, the speed of the $\mathrm{EV}$, and, if possible, the approach lane of the EV at the intersection for more effective control of the preemption phase. Different techniques are used for vehicle detection, including inductive loops, magnetic sensors, microwave radars, IR sensors, ultrasonic sensors, video cameras, radio, and GPS.

Inductive loops are usually installed in the road pavement. They sense the presence of a vehicle by inducing an electric current into the metal of the vehicle. Loop detectors are the most common and reliable method of vehicle's detection, which is used in advanced traffic control systems like SCOOT and SCATS [17]. Magnetic sensors detect the presence of a metallic object (i.e., a vehicle) through the perturbation caused in the earth magnetic field when a vehicle enters the magnetometer's detection zone. Magnetic detectors are installed below the road surface horizontally. They can only detect the passage of a vehicle. Microwave radar's sensors transmit a continuous wave $(\mathrm{CW})$ electromagnetic signals to detect vehicle passage (count), presence and speed of a vehicle. Infrared sensors can be categorized as passive or active and operate in either mode to detect vehicles. Ultrasonic sensors transmit sound waves of frequency above $25 \mathrm{kHz}$ (above human's audible range) and can provide vehicle count, presence, and occupancy. Video-based systems use CCTV cameras and advanced video processing features to detect the presence, speed, and lane occupancy of the vehicles can be measured [18-25]. GPS-based system can effectively determine the vehicle's speed, position, and direction of movement. More recently, the use of magnetoresistive sensors for vehicle's detection and classification was introduced due to its easy installation, low cost, small size, and strong anti-jamming capability [26, 27]. 


\subsection{Impact of preemption on the signal system}

EVs experience heavy delays at intersections due to long queues or stopping at the traffic signal. Previous research investigated different signal preemption strategies to alleviate this problem from the perspective of a single intersection. A study investigated the signal priority problem of EV that was coming from different directions and had to pass the same intersection in the certain time period and presented a signal priority control system for multiple EVs based on multi-agent [28]. A study proposed a degree of priority-based control strategy for EVs to decrease the impacts on general traffic. The performance of the proposed strategy was tested using a microscopic simulation model [29]. Another study proposed two strategies for EV signal preemption, where the first strategy was developed to enable the signal transition from normal operation to EV signal preemption, and the second control strategy was used for the transition from EV signal preemption back to normal operation [8].

Many of the developed preemption strategies operate on a single intersection basis. These kinds of strategies depend on local detection and clear intersection one by one, and signal preemption procedure cannot start until an EV is detected; inherent delays at intersections are unavoidable. This kind of signal preemption strategy, which is based on local detection and clears the intersection one by one, can start only after an EV is detected and usually leads to inevitable intersection delay. Mu et al. proposed a signal preemption control method to reduce time delay of EVs at intersections. According to the time at which $\mathrm{EV}$ is detected and the current phase of each intersection on the traveling route of EV, the calculation methods of the earliest start time and the latest start time of green interval at each intersection were developed [30]. Louati et al. suggested a method favoring the crossing of emergency vehicles (EVs) through intersections in urban cities. They rely on a preemption technique and multi-agent systems. VISSIM traffic simulation software was used for benchmarking and analysis. Several indicators were considered to assess the performance of the network including delay time, travel time, vehicles queue occupancy, number of stops, distance traversed, and speed [7].

Moreover, when multiple signal preemptions are implemented during the peak period, the queue and delay of social vehicles in a given network will be significantly affected [8]. Implementing signal preemption from the perspective of the entire route can reduce the response time of EV. Although substantial progress has been made in signal preemption of isolated intersections, research of dynamic signal preemption based on an entire route is rarely seen. This paper presents a preemption strategy to provide a more efficient route for an EV under a given network and traffic conditions.

Qin and Khan [8] modeled the cost of the path as a function of the distance of the path and its congestion level during at a particular instant of time. One of the main methods used to calculate the shortest path based on the cost defined for the EV to a given destination is the Dijkstra's algorithm [31]. However, the algorithm does not provide any mechanism for collecting real-time traffic data on all routes. Also, it does not address the effect of route selection on signal coordination. The challenge is to locate the incident location immediately that is imperative in this technique. One approach is that the EV operator or the control center operator determines and sets the exact possible location as the destination of the EV and then runs the Dijkstra's algorithm to find the route. Wang et al. [32] proposed an automatic incident location technique is proposed.

Kwon et al. [33] presented an EVP system called Taicang Jiangsu emergency vehicle signal preemption (TJ-EVSP) based on cooperative vehicle-infrastructure system (CVIC). The system used digital short-range communication (DSRC) and $3 \mathrm{G}$ communication to connect the $\mathrm{EV}$ to the general vehicles and with the infrastructure, i.e., traffic signal controllers, field sensors, and control center facility. The EV is equipped with technology to be aware of $* * 360^{\circ}$ of its environment and is fully connected to the infrastructure using communication network and GPS. Field tests were carried out in the city on less than a kilometer road section with a single lane. The results of the TJ-EVSP were compared with the non-preemption (NP) and static preemption scenarios. The average travel time for NP was $107.7 \mathrm{~s}$, while for TJ-EVSP, it was decreased to $65.1 \mathrm{~s}$. One challenge with this system is the high cost of the system, including the hardware installed in $\mathrm{EV}$ and also the need for almost the same hardware for general vehicles, which make the deployment impractical. Fogue et al. [34] proposed control strategies for signal preemption for EV.

The best path can be selected by multiple criteria, e.g., the shortest path by distance, shortest path by the number of intersections and traffic signals, and path that has less traffic at the particular time based on historical data or real-time satellite data or a hybrid method to evaluate the optimal path based on multiple criteria. Once the optimal path is selected, the emergency information is disseminated to other vehicles in the particular path to evacuate the entire route or the approaching lane. The EV path information can be disseminated using vehicle-to-vehicle (V2V) and vehicle-to-infrastructure (V2I) communication. Once the EV clears the intersection, the original cycle and coordination settings (if applicable) are restored. Studies on the route clearance strategy for the EV operation can be found in $[31,32]$.

\section{Methods}

In this study, an effective signal preemption and path selection strategy is proposed. The signal preemption strategy 
aims to alter the control algorithm of the traffic signal controller to clear traffic queue before $\mathrm{EV}$ arrives the intersection and continue on its way without stopping in order to reduce the travel time, whereas the path selection strategy aims to select the best path from the origin of the EV to the destination (i.e., incident location). The best path is considered as the path which is the shortest and has the least possible negative impact on the normal traffic. Furthermore, the proposed algorithm also aims to minimize the impact of the preemption on general traffic (i.e., non-emergency traffic). Thus, it is desired that the preemption strategy is initiated only when required or needed and the controller shall recover immediately after the preemption plan is implemented.

\subsection{Preemption Strategy}

In older preemption systems, the preemption period usually starts when a push button in a fire station or in a remote emergency service control center is activated [8]. Thus, a green wave is created for the EV to pass the fixed corridor. The green wave remains active until the EV passes the intersection by switching off the emergency button. Such kind of manual EVP control cannot be effective in all cases and can cause unnecessary delays for normal traffic. The proposed preemption strategy in this section aims to start the preemption phase at the right time such that neither the EV faces hindrance in the way but the impact of the preemption on the normal traffic is also minimized. To start preemption at the proper time, the proposed logic calculates the minimum detection distance (more accurately, the preemption distance) of EV from the notification period $t$ and the EV operating speed using the following relationship:

$t>=t_{\text {switchover }}+t_{\min }+\mathrm{STI}$

where $t_{\text {switchover }}=$ switchover time of the signal head; $t_{\min }=$ discharge time of the signal, and STI=safety time interval.

The switchover time is the interval of switching the signal state. The discharge time $t_{\min }$ is calculated from the average queue length and the queue discharge speed of the EV approach using historical data. The STI is kept at a constant value of $2 \mathrm{~s}$.

The given values of $t$ and the speed of EV are used to calculate the detection distance. When the EV reaches the position when the $\mathrm{EV}$ is at a distance equal to detection distance (also called as preemption distance), the controller activates the preemption phase by interrupting the normal control phase.

An important parameter is the required green time of the signal head on the EV approach, which can be calculated as:

$t_{g}=t-\left(t_{\text {switchover }}+t_{\min }+\mathrm{STI}\right)$

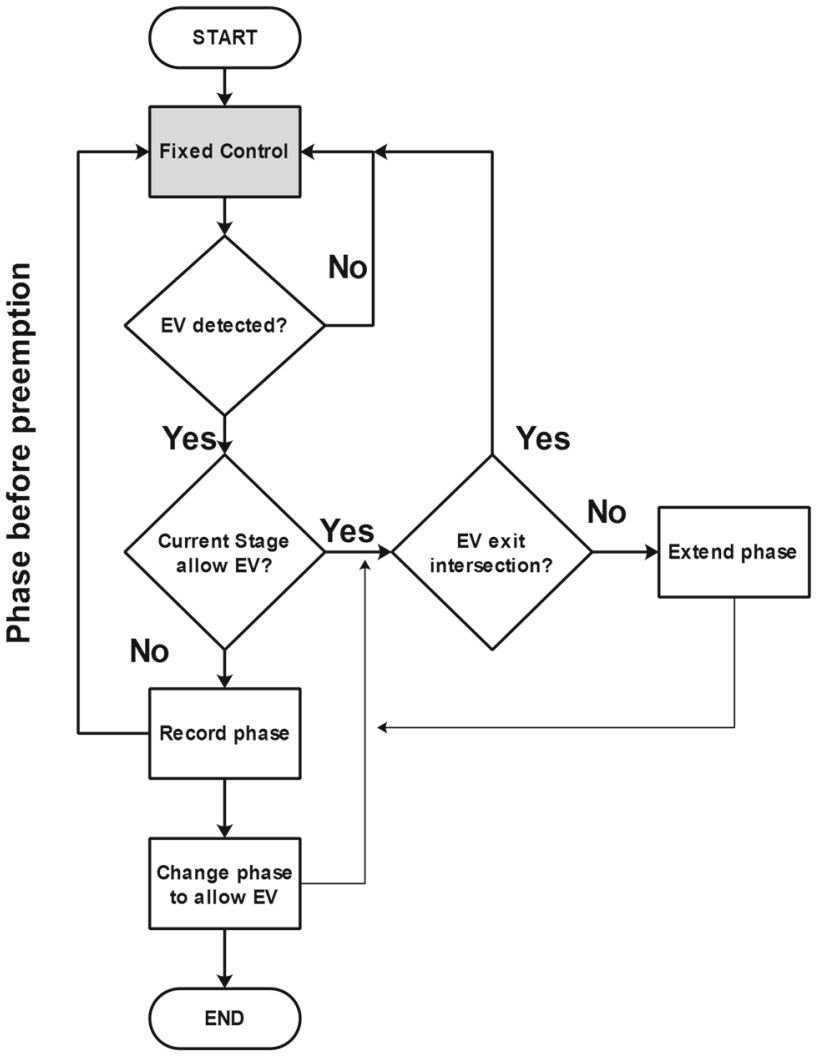

Fig. 1 Flow chart with EV signal preemption

Alternatively, the green time for the preemption phase can be terminated automatically, once the EV is detected to have departed the intersection. The proposed preemption strategy is illustrated in Fig. 1. The controller assumes normal operation by assigning a green interval to the previously active signal head before the preemption phase.

In the case of coordinated route intersections, it is assumed that if the distance between two successive intersections is shorter than the minimum EV detection distance, then the EV detection point for the first intersection will be affected. In this case, the notification period will also account for the discharge time of both intersections. In other words, two intersections will be treated as a single intersection. If the distance between the two consecutive intersections is larger than the detection distance, the detection distance for the second intersection is recalculated similar to the aforementioned approach for a single intersection.

In the next section, the selection of the best path to the incident location is explained.

\subsection{Best-Path Selection}

In EV preemption, the aim is to reduce the response time; thus, the shortest path selection is always critical. It is also expected that the shortest path is heavily congested, but the 
congestion can be cleared by choosing larger detection distance in the preemption stage. Given the location of the EV and incident location, the shortest path from the EV's origin to the incident location is calculated using Dijkstra's algorithm. The road network is modeled so that the roads are represented as links, and the intersections are represented as nodes. The travel cost $T_{(i, k)}$ of the link $i$ during the time interval $k$ is calculated from the length of the link $L_{i}$ and the congestion level $C_{(i, k)}$ during the time $k$ :

$T_{(i, k)}=L_{i}\left(1+C_{(i, k)}\right)$

$C_{i, k}=\sum \frac{\beta_{j}\left(P_{(j, k)} \cdot V_{(j, k)}\right)}{\left(1+V_{(j, k)}\right)}$

where $V_{(j, k)}=$ number of vehicles passed detector $j$ on link $i$ during $k ; B_{j}=$ weight for the detector $j$ in link $i$.

$$
\begin{aligned}
& \sum B_{j}=1 \\
& L_{i}=[0-1] \\
& P_{(j, k)}=\left\{\begin{array}{l}
1: f \text { detector } j \text { is occupied by a vehicle at the end of } k \\
0: \text { otherwise }
\end{array}\right.
\end{aligned}
$$

There are some fundamental requirements in calculating the link cost. First, when the cost of each route is being calculated to find the shortest route, the EV has not yet started its journey. Once the shortest path on the basis of these travel costs is calculated with the Dijkstra's algorithm and the EVs started to follow the shortest path, it is obvious that the link cost will vary each second since more vehicles are passing the detectors. Hence, the proposed scheme takes into account the previous values over the time duration and use regression to predict the vehicle's count. Second, the link cost is not only a function of length and congestion but also of link capacity and other factors such as the number of roundabouts and unsignalized intersections. For simplicity, this study considers signalized intersections only and equal link capacity in the proposed strategy.

In addition to the aforementioned two strategies for signal preemption and path selection in our proposed logic, the accurate estimation of the travel time of the EV along the path can further be integrated within the path planning process. The accurate estimation of the travel time $[35,36]$ depends on several parameters such as link capacity, traffic volume, traffic density, vehicle's composition, link speed limit, average speed of vehicles on the link, cycle length, duration of the effective green cycle, and even the driving behaviors. The parameters related to the traffic signal (e.g., cycle length and green time duration) are only significant for signalized corridors while they become irrelevant for freeways. All other parameters that are mentioned can be used to compute the travel time of vehicles on freeways (i.e., free-flow roads).
The travel time can be used to predict the detection distance in longer road segments.

\subsection{Simulation Environment Test bed}

Due to the critical nature of the EVP problem, it is desired that the proposed scheme should be tested and validated in realistic environments. The proposed scheme is tested in microsimulation software VISSIM, which is widely used for diverse traffic problems [37-39]. VISSIM allows simulating complex vehicle interactions realistically on a microscopic level. The software is built upon the popular car-following model that considers the physical and psychological aspects of the drivers. This simulation package allows the simulation environment to be integrated with external control logic, via the VISSIM-COM interface. The user can develop the controller algorithms or logic flow externally in different formats such as VBA or MATLAB. Then, the simulation environment communicates and transfers data to/from the external controller, in order to interfere with the simulation process and execute the results (which are the preemption actions and signal timing parameters in this case). Consequently, different controller algorithms can be tested and evaluated within the simulation environment without being restricted to the default options or values that are built in within the software [39]. Furthermore, this traffic simulation software is commonly used to investigate problems such as EVP and transit signal priority $[40,41]$.

\subsection{Site Selection and Preparation}

A major arterial with four signalized intersections in the city of Doha, Qatar, was selected for the study (see Fig. 2). The traffic volumes for the three peak hours (morning, midday, and evening) were extracted from the Qatar Strategic Transport Model 2016 (QSTM) (see Fig. 3). Turning movement counts and signal timings were inserted in VISSIM (see Fig. 4). The model was calibrated in order to assure reliable outcomes. The control strategy of the traffic signal controller was implemented as a VAP code in VISSIM.

\section{Results}

\subsection{Emergency Vehicle Preemption Integration within Simulation Environment}

The first transition in preemption starts when an EV is detected at the beginning of the corridor. The VISSIM controller was configured with four signal groups, SG1, SG2, SG3, and SG4 for each intersection. Four stages are defined as Stage_1, Stage_2, Stage_3, and Stage_4. Each stage is assigned to a single group in ascending order, (e.g., SG1 
Fig. 2 Aerial image for the major arterial

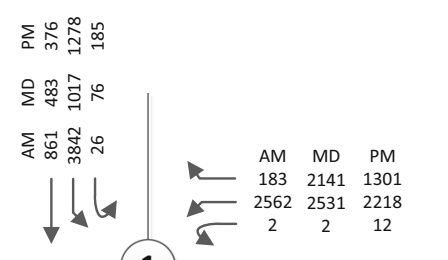

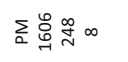

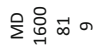

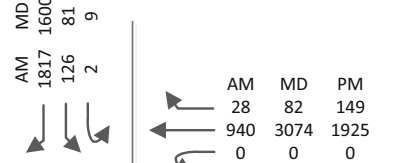

엄

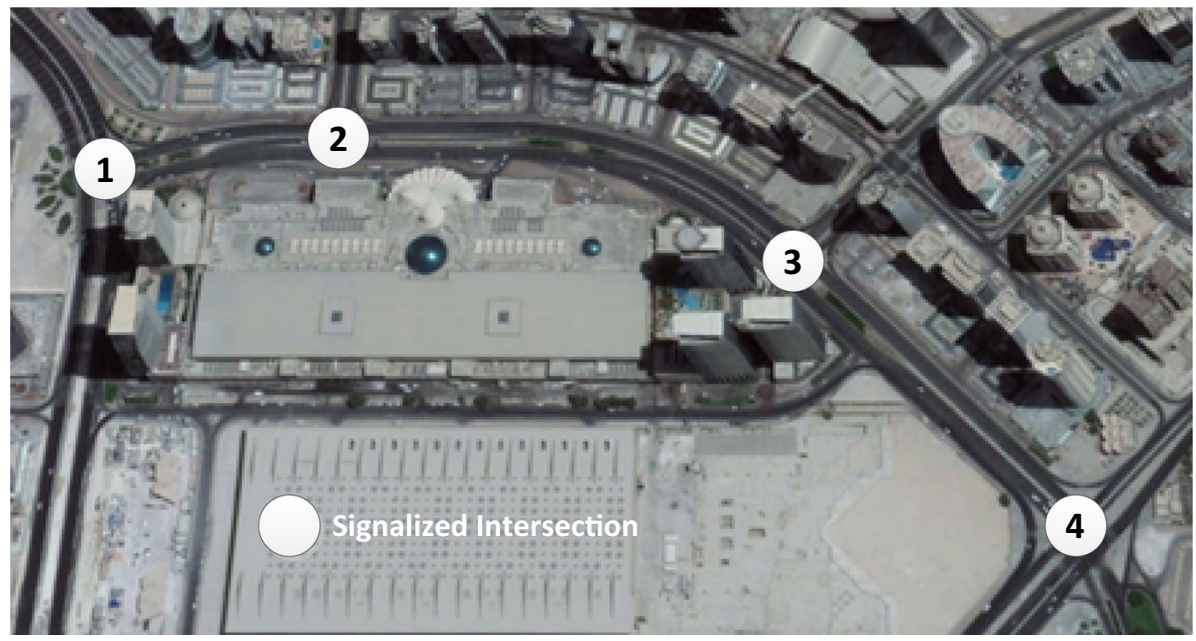

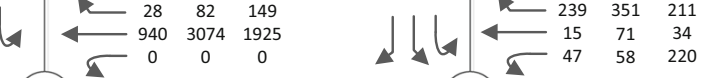

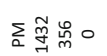

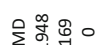

$\sum_{<}^{\infty}$
ำลำำ

这守需的

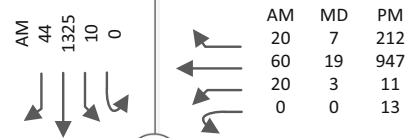

(1)

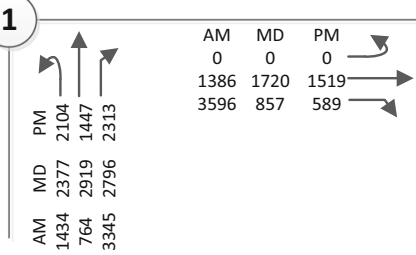

\section{2 (AM $M{ }^{2} P M \quad-308$}

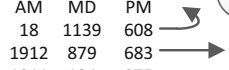

$\begin{array}{lll}1912 & 879 & 683 \longrightarrow \\ 1911 & 194 & 375\end{array}$

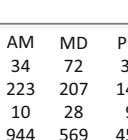

$\begin{array}{ccc}223 & 207 & 140 \\ 10 & 28 & 9 \\ 944 & 569 & 458\end{array}$

4

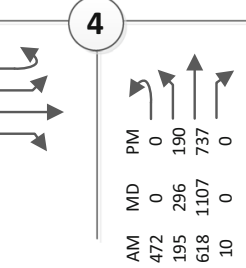

Fig. 3 Traffic volumes for AM, MD, and PM peak hours

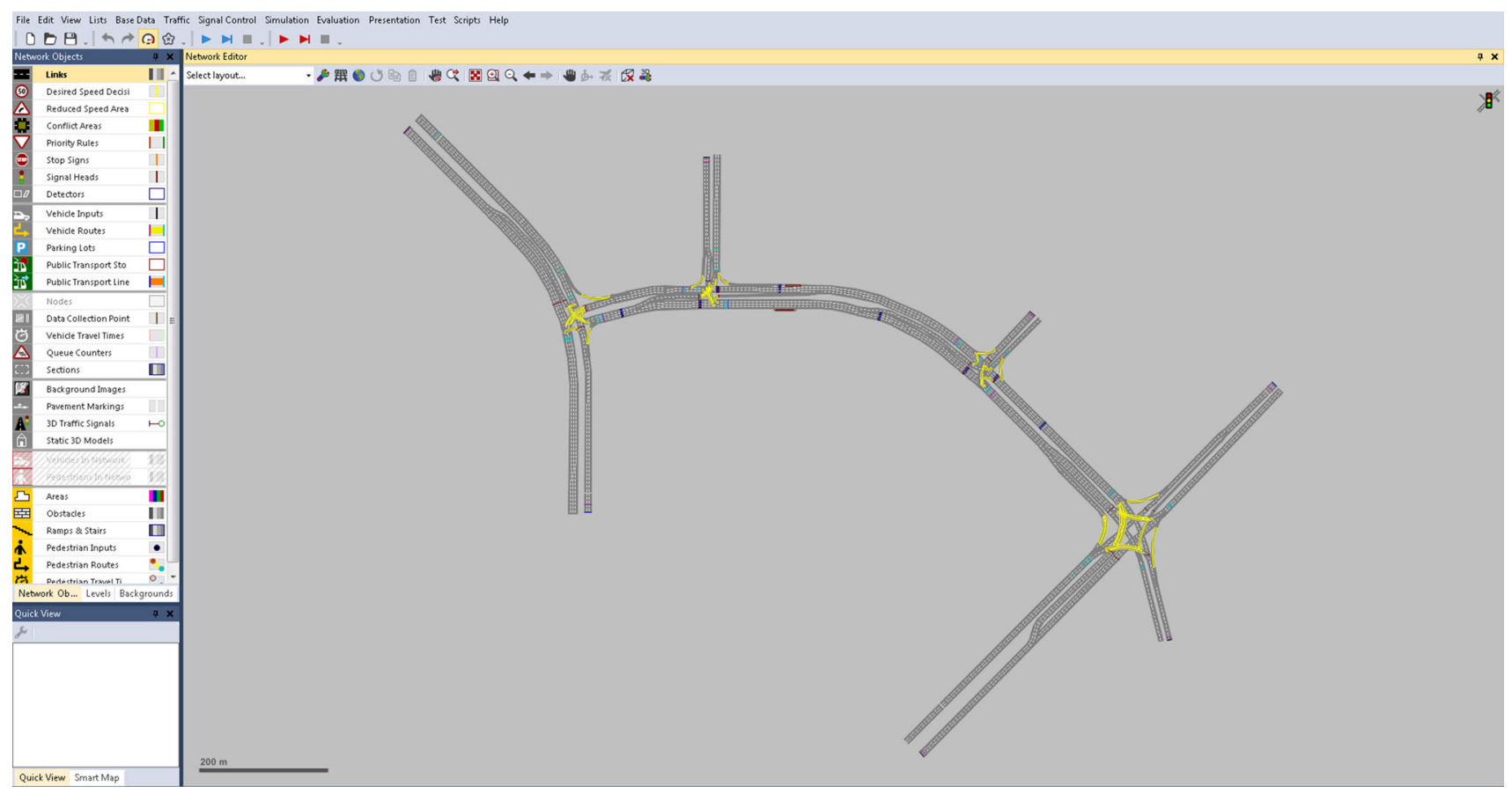

Fig. 4 Traffic network in VISSIM simulation environment 
is assigned to Stage_1 and so forth). For the purpose of EV detection, detectors were positioned along the arterial. The algorithm of the proposed signal group-based controller implemented in VISSIM is shown in Fig. 1. Two strategies were used in the traffic signal controller algorithm, early green and green extension. In the case of early green, the red time is truncated and switched to green by the anticipated arrival time of the EVs. In the case of green extension strategy, an extension was given to the regular green time if the $\mathrm{EV}$ is predicted to reach the intersection within a short duration beyond the actual green time and before the next green interval. As soon as the EV is detected to pass the intersection, the preferential treatment of extended green is terminated.

The corridor was first simulated with traffic control without the preemption strategy. Next, the corridor was simulated with preemption control logic to see the impact of preemption. In this case, a special vehicle EV was added to the scenario using VISSIM-COM interface for a controller logic that was created in MATLAB environment. The EV entered the network through the eastbound link entering, with a maximum speed of $80 \mathrm{~km}$ per hour. When the EV is detected at the designated check-in detectors installed in the pavement, a signal is transmitted to the signal controller. The signal controller then initiates the preemption stage immediately. Once the EV exits the intersection and gets detected on one of the three sets of check-out detectors, a second signal is transmitted to the signal controller that terminates the preemption stage, and a transition to normal control stage is started.

\subsection{Simulation Results}

The proposed preemption strategy was evaluated using VISSIM for three different peak periods. The results were summarized to show the impact of the preemption strategy on the EVs and general traffic. As shown in Fig. 5, the results clearly showed a considerable decrease in the travel time for EVs. These results are expected since the preemption logic is designed to benefit EVs in the form of a reduction in delay, number of stops, and travel time. EVs observed a decrease in the travel time that varied between 15.8 and $48.8 \%$, for different traffic conditions. The results also indicated that the EVs benefited the most in the case of lower traffic volumes, which is expected. The simulation results exhibit the advantage of preempting strategy. The average travel time for the EV was reduced by $24.3 \%$ using preemption strategy.

Furthermore, the average delay per vehicle was calculated at each intersection and indicated a general delay reduction in most cases as shown in Fig. 6. These results can be explained by the fact that the general traffic in the direction of the EV benefited the most from the preemption because the vehicles in this direction receive additional green time during preemption. Other approaches are expected to have different levels

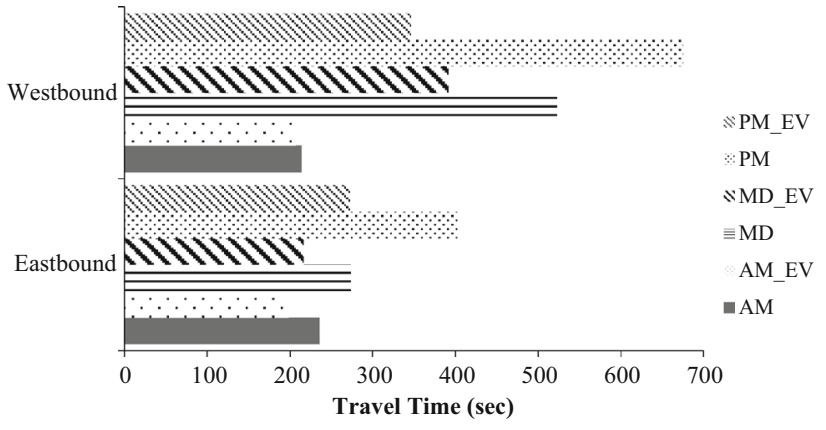

Fig. 5 Travel time of eastbound and westbound directions under different peak hours with and without preemption

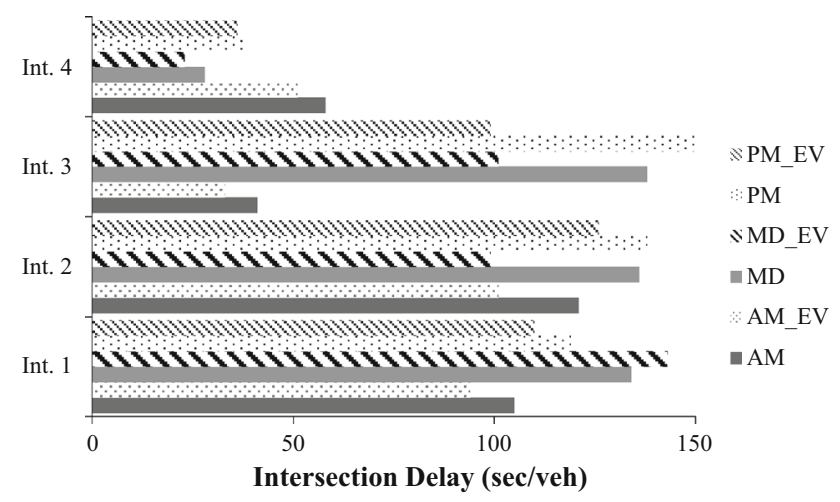

Fig. 6 Average intersection delay under different peak hours with and without preemption

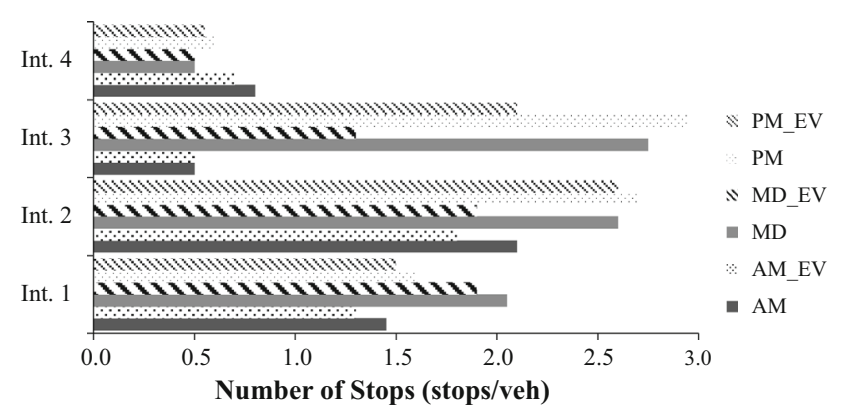

Fig. 7 Average number of stops under different peak hours with and without preemption

of negative impact. In general, the main directions of the arterial experience lower delays and better progression when compared to the crossing streets. Some scenarios showed a delay reduction that surpassed $35 \%$. However, in most cases, traffic delay was reduced. Nonetheless, there are cases where some intersections showed marginally higher delay. This is attributed to the reduction in green time and losing progression. Similar results were obtained for the average number of stops per vehicle (Fig. 7). In some cases, the number of stops was reduced by over $50 \%$. 


\section{Conclusion}

This study presented and evaluated the potential benefits of implementing a proposed preemption strategy along a major corridor. The results indicated a significant benefit for the EVs after implementation of preemption. For general traffic, the results varied. In most cases, the general traffic, especially along the mainstream, benefited from the implementation of preemption due to receiving more green times and improved progression. In other cases, the results showed a marginally negative impact. The negative impact of preemption on general traffic can be significantly reduced by the fast transition of the signal phases after the EV exits the intersection and the fair distribution of green time immediately after preemption phase can minimize the negative impact of preemption. While the study shows that the proposed strategy could benefit both EVs and general traffic, the results should not be considered as a general rule for evaluating the preemption effect. Several factors can affect the results such as the traffic volumes, signal timings, the spacing between intersections, and type of facility. The outcome of this research provides a strategy that can give an indication of the impact of preemption and can provide a process useful for the evaluation of preemption along traffic networks.

\section{Discussion}

The older schemes for EV preemption were based on manual activation of preemption phase using an emergency button turned on at the EV origin or a control center. These manual schemes although provide a green wave to the EV along the route to reach the incident location: however, it adversely impacts the normal traffic along the corridor. To avoid the impact of EVP over normal traffic, the proposed scheme aims to activate the preemption phase at the right time. The proposed scheme calculates the minimum detection distance based on the actual traffic conditions along the EV route and triggers the traffic controller to activate the preemption phase when the EV reaches this location. Additionally, the proposed scheme incorporates a path selection strategy. The shortest path is selected using Dijkstra's algorithm before the EV is departed from the origin. Once the optimal path is selected, the emergency information can be disseminated to other vehicles in the particular path to evacuate the entire route or the approaching lane, which is a major advantage compared to other methods. The EV path information can be disseminated using vehicle-to-vehicle (V2V) and vehicle-to-infrastructure (V2I) communication in the particular path to evacuate the entire route or the approaching lane. Once the EV clears the intersection, the original cycle and coordination settings (if applicable) are restored.
As future research, the proposed preemption strategy should be implemented and evaluated using real-time traffic data. Moreover, the impact of multiple simultaneous preemptions should be investigated. A common case is when one EV is followed by another with a distance between them, and the first $E V$ exits the intersection before the notification from the second EV is received. The recovery phase will be disturbed with the second preemption, and the fair distribution of the green time becomes more difficult. Another case which is even worse is the two simultaneous preemptions on two different and conflicting approaches of the same intersection. This kind of situation can arise in extreme emergency cases. Finally, the design of robust traffic signal controllers and reliable detection systems for both vehicles and incidents are the upcoming challenges in this field, while neural networks, advanced genetic algorithms, and machine learning are the candidate technologies to cope with these challenges.

Acknowledgements Open access funding provided by the Qatar National Library.

Open Access This article is distributed under the terms of the Creative Commons Attribution 4.0 International License (http://creativecomm ons.org/licenses/by/4.0/), which permits unrestricted use, distribution, and reproduction in any medium, provided you give appropriate credit to the original author(s) and the source, provide a link to the Creative Commons license, and indicate if changes were made.

\section{References}

1. Paniati, J.F.; Amoni, M.: Traffic signal preemption for emergency vehicles. In: RITA. US Development of Transportation Research and Innovative Technology Administration (2006).

2. Mirchandani, P.B.; Lucas, D.E.: Integrated transit priority and rail/emergency preemption in real-time traffic adaptive signal control. J. Intell. Transport. Syst. 8(2), 101-115 (2004)

3. Huang, Y.-S.; Weng, Y.-S.; Zhou, M.: Design of traffic safety control systems for emergency vehicle preemption using timed Petri nets. IEEE Trans. Intell. Transport. Syst. 16(4), 2113-2120 (2015)

4. Younes, M.B.; Boukerche, A.: An efficient dynamic traffic light scheduling algorithm considering emergency vehicles for intelligent transportation systems. Wireless Netw. 24(7), 2451-2463 (2018)

5. Marcianò, F.A.; Musolino, G.; Vitetta, A.: Signal setting optimization on urban road transport networks: the case of emergency evacuation. Saf. Sci. 72, 209-220 (2015)

6. Kimpel, T.J.; Strathman, J.; Bertini, R.L.; Callas, S.: Analysis of transit signal priority using archived TriMet bus dispatch system data. Transport. Res. Rec. 1925(1), 156-166 (2005)

7. Louati, A.; Elkosantini, S.; Darmoul, S.; Louati, H.: Multi-agent preemptive longest queue first system to manage the crossing of emergency vehicles at interrupted intersections. Eur. Transp. Res. Rev. 10(2), 52 (2018)

8. Qin, X.; Khan, A.M.: Control strategies of traffic signal timing transition for emergency vehicle preemption. Transport. Research Part C: Emerg. Technol. 25, 1-17 (2012)

9. Sims, A.G.; Dobinson, K.W.: The Sydney coordinated adaptive traffic (SCAT) system philosophy and benefits. IEEE Trans. Veh. Technol. 29(2), 130-137 (1980) 
10. Hunt, P.B.; Robertson, D.I.; Bretherton, R.D.; Royle, M.C.: The SCOOT on-line traffic signal optimisation technique. Traffic Eng. Control 23(4), 190-192 (1982)

11. Mirchandani, P.; Wang, F.Y.: RHODES to intelligent transportation systems. IEEE Intell. Syst. 20(1), 10-15 (2005)

12. Ghamann, R.; Gettman, D.; Shelby, S.: ACS lite project overview. In: TRB Adaptive Traffic Signal Control Workshop, Washington, DC (2004).

13. Opticom Emergency Vehicle Preemption (EVP): GTT. http://www. gtt.com/opticom-emergency-response/. Accessed 18 Mar 2019

14. Chadwick, C.: BLISS: Brisbane's linked intersection signal system-co-ordinating the city's traffic signals using a personal computer. In: Local Government Engineers' Association of Queensland, Annual Conference, 1992, Ipswich, Queensland, (1992)

15. STROBECOM II: https://www.tomar.com/products/trafficcontrol/strobecom-ii/. Accessed 18 Mar 2019

16. The Mirt Traffic Light Control Device: http://www.themirt.com/ index.php. Accessed 18 Mar 2019

17. Luk, J.: Two traffic-responsive area traffic control methods: SCAT and SCOOT. Traffic Eng. Control 25(1), 14 (1984)

18. Gettman, D.M.: A multi-objective integrated large-scale optimized ramp metering control system for freeway/surface-street traffic management (1998).

19. Gradinescu V.; Gorgorin, C.; Diaconescu, R.; Cristea, V.; Iftode, L.: Adaptive traffic lights using car-to-car communication. In: Spring. IEEE 65th Vehicular Technology Conference, 2007 (VTC2007), pp. 21-25. IEEE (2007)

20. Zhang, W.; Wu, Q.J.; Yang, X.; Fang, X.: Multilevel framework to detect and handle vehicle occlusion. IEEE Trans. Intell. Transport. Syst. 9(1), 161-174 (2008)

21. Zhang, W.; Wu, Q.J.; Yang, X.; Fang, X.: Multilevel framework to detect and handle vehicle occlusion. IEEE Trans. Intell. Transport. Syst. 9(1), 161-174 (2008)

22. Buch, N.; Orwell, J.; Velastin, S.A.: Urban road user detection and classification using 3D wire frame models. IET Comput. Vision 4(2), 105-116 (2010)

23. Cheng, H.Y.; Weng, C.C.; Chen, Y.Y.: Vehicle detection in aerial surveillance using dynamic Bayesian networks. IEEE Trans. Image Process. 21(4), 2152-2159 (2011)

24. Robert, K.: Video-based traffic monitoring at day and night vehicle features detection tracking. In: 12th International IEEE Conference on Intelligent Transportation Systems, 2009 (ITSC'09), pp. 1-6. IEEE (2009)

25. Li, Y.; Li, B.; Tian, B.; Yao, Q.: Vehicle detection based on the and-or graph for congested traffic conditions. IEEE Trans. Intell. Transport. Syst. 14(2), 984-993 (2013)

26. Nguyen, V.D.; Nguyen, T.T.; Nguyen, D.D.; Lee, S.J.; Jeon, J.W.: A fast evolutionary algorithm for real-time vehicle detection. IEEE Trans. Veh. Technol. 62(6), 2453-2468 (2013)
27. Taghvaeeyan, S.; Rajamani, R.: The development of vehicle position estimation algorithms based on the use of AMR sensors. IEEE Trans. Intell. Transport. Syst. 13(4), 1845-1854 (2012)

28. Ma, C.; Cui, J.: Multiple emergency vehicles signal priority control based on multi-agent approach. J. Transport. Syst. Eng. Inf. Technol. 13(1), 57-62 (2013)

29. Wang, J.; Ma, W.; Yang, X.: Development of degree-of-priority based control strategy for emergency vehicle preemption operation. Discrete Dynamics in Nature And Society, vol. 2013 (2013).

30. Mu, H.; Liu, L.; Li, X.: Signal preemption control of emergency vehicles based on timed colored petri nets. Discrete Dynamics in Nature and Society, vol. 2018 (2018).

31. Pandit, K.; Ghosal, D.; Zhang, H.M.; Chuah, C.-N.: Adaptive traffic signal control with vehicular ad hoc networks. IEEE Trans. Veh. Technol. 62(4), 1459-1471 (2013)

32. Wang, J.; Yun, M.; Ma, W.; Yang, X.: Travel time estimation model for emergency vehicles under preemption control. Procedia-Social and Behavioral Sciences 96, 2147-2158 (2013)

33. Kwon, E.; Kim, S.; Betts, R.: Route-based dynamic pre-emption of traffic signals for emergency vehicle operations. In: Proceedings of the TRB Annual Meeting, vol. 9: Transportation Research Board Washington, DC, USA (2003).

34. Fogue, M.; Garrido, P.; Martinez, F.J.; Cano, J.-C.; Calafate, C.T.; Manzoni, P.: A system for automatic notification and severity estimation of automotive accidents. IEEE Trans. Mob. Comput. 13(5), 948-963 (2014)

35. Marshall, S.V.: Vehicle detection using a magnetic field sensor. IEEE Trans. Veh. Technol. 27(2), 65-68 (1978)

36. Kwon, E.; Kim, S.: Development of dynamic route clearance strategies for emergency vehicle operation. Phase 1, Center for Transportation Studies University of Minnesota, Minneapolis, USA (2003)

37. Ghanim, M.S.; Shaaban, K.: A case study for surrogate safety assessment model in predicting real-life conflicts. Arab. J. Sci. Eng. 44(5), 4225-4231 (2019)

38. Kim, I.; Larue, G.; Ferreira, L.; Rakotonirainy, A.; Shaaban, K.: Traffic safety at road rail level crossings using a driving simulator and traffic simulation. Transp. Res. Rec.: J. Transport. Res. Board 2476, 109-118 (2015). https://doi.org/10.3141/2476-15

39. Shaaban, K.; Kim, I.: Comparison of SimTraffic and VISSIM microscopic traffic simulation tools in modeling roundabouts. Proc. Comput. Sci. 52, 43-50 (2015). https://doi.org/10.1016/j.procs. 2015.05.016

40. So, J.; Krause, S.; Kaths, J.; Tian, L.; Moon, Y.J.: A proactive emergency vehicle control strategy based on automated driving technologies (2018).

41. Shaaban, K.; Ghanim, M.: Evaluation of transit signal priority implementation for bus transit along a major arterial using microsimulation. Proc. Comput. Sci. 130, 82-89 (2018) 